\title{
Pathophysiology of ischaemic heart disease
}

Authors: Mamatha Oduru Pocock, Lucy Dorrell, Paola Cicconi

Affiliations:

Nuffield Department of Medicine and Oxford NIHR Biomedical Research Centre, University of

Oxford, OX3 7FZ

Conflicts of interest: No conflicts of interest declared from all three authors.

Word count:

Abstract: 193 words

Text: 2475 


\section{KEY POINTS}

- Ischemic Heart Disease (IHD) in PLWH is still a major cause of non-AIDS related mortality, however the incidence of this condition is declining. Recent findings suggest that primary prevention measures, such as smoking cessation, prescription of lipid-lowering drugs and diet and exercise modifications, may be instrumental in this reduction.

- The benefit of virological suppression on CVD morbidity and mortality is particularly evident in individuals with a low nadir CD4 and high prevalence of metabolic risk factors.

- The association between abacavir exposure and myocardial infarction (MI) is still contentious and a biological mechanism has yet to be identified. However, the association between abacavir and MI was re-confirmed in a recent analysis performed by the DAD Study Group which importantly discounted channelling bias as an explanation for this finding.

- In the era of modern therapy, there has been a move away from solely focusing on traditional risk factors and ART induced metabolic changes; underlying pathological processes such as inflammation, atherosclerosis and endothelial dysfunction are gaining significance.

- Virologically suppressed individuals on modern ART appear to have significant background inflammation, higher incidence of vulnerable atherosclerotic plaque features and endothelial dysfunction. Recent research hypothesises that these pathological processes contribute significantly to increased IHD risk in PLWH. 


\section{Abstract}

Purpose of review: to summarize recent findings in the pathogenesis of ischemic heart disease (IHD) in people living with HIV (PLWH).

Recent findings: People living with HIV (PLWH) have an elevated risk of ischemic heart disease (IHD). Although incidence is declining, this condition still represents a major cause of non-AIDS related mortality. The aetiology is likely multifactorial: traditional risk factors play an important role and IHD risk might be reduced with greater emphasis on primary prevention. The contribution of specific antiretroviral agents to IHD risk is changing as antiretroviral coverage increases globally and as safer agents have replaced drugs with well-described metabolic toxicities. The beneficial impact of virological suppression on ART in reducing IHD is particularly evident in participants with advanced HIV infection and high baseline cardiovascular risk. The association between current abacavir use and myocardial infarction is still unexplained and indicates that mechanisms other than metabolic alterations may underlie IHD in PLWH. Consequently, the contributions of inflammation, sub-clinical atherosclerosis and endothelial dysfunction are receiving greater attention.

Summary: Modern ART coupled with intensified efforts towards primary prevention is the cornerstone of IHD risk management in PLWH. The role of chronic inflammation and its optimal management need to be defined. 


\section{INTRODUCTION}

With increasing life expectancy and the chronicity of HIV infection, cardiovascular disease (CVD) has emerged as an important cause of morbidity and mortality in PLWH. In developed countries, the most common cardiovascular complication of HIV is ischaemic heart disease (IHD), comprising stable and unstable angina, myocardial infarction (MI) and sudden cardiac death. HIV infection carries a $50 \%$ increased risk of acute myocardial infarction compared to HIV negative controls after adjusting for Framingham risk factors, comorbidities, and substance abuse [1]. According to the Data Collection on Adverse Effect of Anti-HIV Drugs (DAD) study, CVD is the fourth most common cause of death in PLWH, with $\mathrm{MI}$ accounting for $6 \%$ of total mortality in Europe and the United States (US) [2]. Emerging data from Sub Saharan Africa (SSA) are scarce and are mostly derived from cross-sectional studies but also suggest that PLWH have an increased CVD risk [3][4].

The underlying pathology of IHD in HIV infection includes atherosclerosis and vascular abnormalities such as endothelial activation and dysfunction. The aetiology is likely multi-factorial. PLWH are known to have a high prevalence of traditional risk factors, for example, prevalence of smokers in European and US cohorts was 60\% [5]. Metabolic side effects of first-generation antiretroviral agents are well documented. HIV-related immune activation and chronic inflammation have been proposed as an explanation for the excess IHD risk that persists after controlling for these factors. As modern ART reduces the likelihood of drug-induced metabolic syndromes and enables sustained virological control in adherent patients, elucidating the core pathological mechanisms such as inflammation, subclinical atherosclerosis and endothelial dysfunction is now a priority. In this review we discuss recent findings that are beginning to refine our understanding of the pathogenesis of IHD in PLWH.

\section{TRADITIONAL RISK FACTORS}

Recent epidemiological data suggest that there has been a decline in CVD- and IHD-associated mortality in PLWH [2] [6][7] and highlight the crucial role of modifiable traditional risk factors. In the DAD study, the observed decrease in CVD-related deaths over time could not be explained by the higher proportion of virologically suppressed individuals, suggesting that deployment of primary prevention measures such as smoking cessation, prescription of lipid-lowering drugs, diet and exercise modifications may be responsible [2]. This hypothesis was supported by a recent study that assessed the short-term mortality after MI in the same cohort [6]. It confirmed that prescription of lipid-lowering drugs and anti-platelet agents resulted in significantly improved survival rates, despite the older age and poorer CVD risk profile of in individuals who experienced an MI. This study 
highlights the importance of aggressive secondary preventive measures post-MI in PLWH, as well as the need to continue efforts to reduce CVD risk.

In a large American cohort of insured patients, HIV-positive individuals had a similar risk for IHD in 2010-2011 compared with a matched control of HIV-negative individuals in contrast to previous studies describing higher risk of myocardial infarction among PLWH during the early ART era [1][7] [8]. Furthermore, trends in the use of lipid-lowering and anti-hypertensive therapy among HIVpositive and negative subjects were similar. Although these findings from a large integrated care setting of insured individuals cannot be generalized to the overall population of PLWH, they support the concept that increased IHD risk might be, at least partially, reversible with emphasis on primary prevention.

Dyslipidemia, hypertension, anti-aggregants and tobacco use are discussed in more detail in accompanying reviews in this issue of the journal.

\section{THE IMPACT OF ANTIRETROVIRAL THERAPY (ART)}

Associations between specific antiretroviral agents and metabolic alterations leading to increased IHD risk are well described in different care settings [8][9][10][11][12][13]. On the other hand, it has become clear that effective ART reduces risk by suppressing viral replication and restoring immune function. However, the START trial [14], an intercontinental randomised controlled study to assess the effect of immediate treatment in asymptomatic HIV infected individuals, did not show a difference in cardiovascular event rate did according to timing of ART initiation. This may reflect the very low rate of CVD events overall (1.9/1000 person-years among 4685 participants over a mean 3 years' follow-up) and the relative immune preservation and short duration of diagnosed HIV infection. Similarly, the HPTN-052 study, another randomised trial, failed to demonstrate the protective role of earlier (CD4 count $>350$ ) versus late ART initiation on CVD, most likely because of the short duration of follow-up (median 2 years) [15].

Much of the data demonstrating greater CVD risk at lower CD4 counts and higher HIV viral loads factors that improve with ART - derive from studies of participants with more advanced HIV disease and higher baseline CVD risk [1][16]. A recent retrospective cohort study of 2960 PLWH in Brazil demonstrated the overall benefit of ART on CVD morbidity and mortality in individuals with a low nadir CD4 and high prevalence of metabolic risk factors [17]. In this population, detectable HIV viral load in the prior year and nadir CD4 of $\leq 50$ cells/ $\mu$ l were independently associated with CV events, 
after adjustment for traditional risk factors. The authors also examined associations between antiretroviral drug class and the risk of CVD events. Cumulative NRTI, NNRTI, and/or PI exposure were associated with a 12-23\% decreased CV event incidence per additional year of exposure, with recent tenofovir and atazanavir use having the strongest effects. As this was a non-randomised retrospective study, interpretation is limited by potential treatment selection bias. However, it supports the evidence of a more favourable impact on CVD risk of newer drugs.

The association between abacavir exposure and myocardial infarction (90\% increase in risk, which was first reported in the DAD study in 2008) is still contentious and a biological mechanism has yet to be identified [18]. The DAD study group conducted new research in order to describe subsequent changes in prescribing patterns of abacavir, since the original study was confounded by treatment selection bias (the drug was preferentially prescribed to those with underlying CVD risk in order to avoid older NRTIs with known adverse lipid effects) [19]. Since 2008, those with a moderate to high CVD risk were more likely to discontinue abacavir regardless of viral load; no such pattern was seen previously. After adjustment for potential confounders, the association between abacavir and MI was once again confirmed, with a calculated $98 \%$ increased risk of MI. Hence the finding of a raised risk of MI for people on abacavir is unlikely explained by channelling bias. These results are of particular importance at a time when the use of abacavir, is likely to increase within many settings due to the availability of generic co-formulations and single tablet regimens.

\section{THE ROLE OF INFLAMMATION}

Chronic inflammation causes elevated circulating levels of C-reactive protein (CRP), chemokines and cytokines, coagulation abnormalities, endothelial dysfunction (ED) and premature atherosclerosis with features of plaque vulnerability [20]. Research in the general population now recognises inflammation as a key mediator in the pathogenesis of IHD [21]. Uncontrolled HIV replication is characterised by immune activation, particularly during primary infection, which diminishes but does not disappear with long term virological suppression on ART [22][23]. This was highlighted in the Strategies for Management of Antiretroviral Therapy (SMART) study which demonstrated excess mortality in patients randomised to intermittent therapy compared to daily therapy; this was attributed to elevated HIV-induced inflammation and a pro-thrombotic state in poorly controlled infection [24]. Consequently, there has been a shift in research focus away from traditional risk factors and antiretroviral toxicity towards understanding the role of HIV-induced inflammation in the pathogenesis of CVD in PLWH. 
Recently, the association between inflammation and cardiovascular morbidity and mortality has been explored in HIV cohorts. A systematic review found that CRP, interleukin-6 (IL-6) and D-dimer levels were associated with increased CVD prevalence, which is consistent with findings in the general population [25]. In an exploratory analysis of patients participating in three international HIV treatment trials (SMART, ESPRIT, SILCAAT) who experienced fatal or non-fatal cardiovascular events, IL-6 and D-Dimer levels were associated with mortality at baseline and during follow-up [26].

As CVD endpoint studies require many years of follow-up, a causal relationship between inflammatory mediators and CVD has largely been inferred from studies examining surrogate markers of IHD such as subclinical coronary artery disease. An analysis of $>900$ participants in the Multicenter AIDS Cohort (575 HIV-positive and 348 HIV-negative men) found that higher levels of IL6 and intracellular adhesion molecule 1 (ICAM-1) were associated with greater prevalence of moderate to severe ( $>50 \%$ ) coronary artery stenosis on computed tomography angiography (CTa) in HIV-positive participants; these associations remained significant after adjustment for traditional cardiovascular risk factors and HIV clinical factors (nadir CD4 count, viral load, duration of infection [27]. High levels of inflammatory markers were associated with more advanced atherosclerosis (coronary stenosis and coronary artery calcification) and thus were considered to be a reliable indicator of potentially clinically significant vessel disease.

At the present time, the utility of inflammatory biomarkers in predicting IHD risk is uncertain and needs to be substantiated by prospective studies that include clinical endpoints, in order to assess their reproducibility and specificity, since they are not specific for cardiovascular injury. In addition, these biomarkers need to be evaluated further in diverse ethnic groups and in women. Nonetheless, this is an important avenue of investigation when considering the potential for risk stratification in high-risk individuals.

\section{ATHEROSCLEROSIS AND PLAQUE CHARACTERISTICS}

Atherosclerosis results from low density lipoprotein (LDL) deposition in the intima of medium- and large-sized arteries, combined with hardening of arterial walls. Vulnerable sections of plaque have the potential to break off and cause thrombotic occlusion, which may result in IHD, stroke or peripheral vascular disease. In the HIV-negative population, complex inflammatory and biomechanical processes promote vulnerable plaque features; on computed tomography angiography (CTa), these appear as positively remodelled and calcified plaques with a higher plaque 
burden [28]. The development of these features is strongly correlated to metabolic risk factors such as hypercholesterolaemia and impaired glucose tolerance.

Non-invasive imaging techniques such as carotid intima-media thickness (CIMT) and coronary CTa now allow researchers to visualise pathological processes in patients before clinical events occur, thus serving as a surrogate marker for future risk. CIMT is a reflection of subclinical atherosclerosis and is thought to correlate with atherosclerosis in other vascular areas, such as the coronary vessels. A cross-sectional study in a well-established cohort in the Netherlands found greater CIMT in HIVpositive individuals compared to age- and gender-matched reference values [29]. However, traditional CVD risk factors were more strongly associated with CIMT than HIV-specific factors, although current ART use showed an inverse correlation. Similar observations were made in studies of CIMT in HIV-infected, mainly female subjects in rural South Africa [30].

Emerging data from studies using CTa suggest that the pathogenesis may differ in PLWH: noncalcified plaques appear to be more common and it is hypothesised that plaque vulnerability in HIV is caused by chronic inflammation rather than the total physical burden of atherosclerosis alone [31]. Furthermore, non-calcified plaque rates were inversely related to CD4 counts, which could explain the association between immunosuppression and increased IHD risk. A retrospective analysis of PLWH without a history of coronary artery disease who underwent coronary CTa not only confirmed previous reports of greater non-calcified plaque burden but also reported a higher rate of non-ST elevation acute coronary syndromes [32]. Collectively, these data suggest that coronary CTa could be a valuable tool in the assessment of IHD risk in asymptomatic HIV-positive patients, though this requires further evaluation in prospective studies.

\section{ENDOTHELIAL DYSFUNCTION}

Vascular endothelium lines all blood vessels and is central to effective coagulation, angiogenesis and regulation of blood flow. Endothelial dysfunction (ED) is a precursor for atherosclerosis and an important contributor to IHD in PLWH and the general population [33]. ED reduces the ability of vessels to dilate in response to nitric oxide; the resulting loss of vaso-reactivity increases the risk of ischaemia [34]. The pro-inflammatory state in HIV infection is thought to exacerbate ED in both treated and untreated patients as inflammation causes activation of endothelial cells, leading to release of cytokines and chemokines (such as IL-6 and monocyte chemoattractant protein, MCP-1) which may play a key role in initiating atherosclerotic plaque development [24][35]. 
Numerous methods have been utilised to measure early ED and include endogenous biomarkers (Asymmetric Dimethylarginine, ADMA), flow-meditated ultrasound, invasive catheterisation techniques and non-invasive MRI imaging. In addition, systemic inflammatory markers and brachial arterial flow have been widely used, although it is unknown to what extent indicators of peripheral ED can be generalised to coronary artery dysfunction. lantorno et al. compared endothelial function in HIV-positive and negative subjects with and without coronary artery disease (CAD) in the largest study of its kind [36]. Ninety-five percent of the HIV-positive cohort was virologically suppressed and traditional risk factors were similar between the two groups. Using isometric handgrip exercises, a non-invasive MRI technique quantified coronary flow velocity and blood flow to measure vasoreactivity and thus detect ED. The study demonstrated that HIV-positive virologically suppressed patients with no significant coronary atherosclerosis had comparable coronary ED to that of HIVnegative subjects with established CAD. An underlying inflammatory hypothesis was suggested as circulating IL-6 levels were significantly higher $(p<0.001)$ in HIV-positive patients without CAD compared to HIV-negative controls without CAD. The above findings need to be substantiated by prospective studies as this study only provided cross-sectional data. However, the extent of ED in PLWH with well-controlled infection on modern ART is noteworthy and may this be a useful clinical predictor of CAD in the future.

\section{CONCLUSIONS AND FUTURE DIRECTIONS}

The beneficial effects of early ART on immune function enable PLWH to reach old age; management of IHD risk is therefore assuming greater importance in their long-term care. The decline in incidence of IHD in PLWH in recent years, despite a high prevalence of smoking, reflects greater appreciation of the contributions of specific antiretroviral agents, metabolic risk factors, uncontrolled HIV replication and associated immune activation to the pathogenesis of IHD, together with growing recognition among clinicians and patients that these risks need to be better quantified and mitigated. However, the mechanisms underlying differences in atherosclerotic plaque development and endothelial dysfunction in HIV infection are not precisely defined and we still lack critical information regarding the optimal management of IHD risk in asymptomatic PLWH on effective ART regimens. A randomised controlled trial suggested that statin therapy may reduce high-risk plaque burden in PLWH; long-term follow-up to assess impact on CVD events is needed [37] [38]. Finally, much of our knowledge to date is derived from studies in industrialised countries and may not be generalisable to SSA, where $70 \%$ of the world's HIV-positive population live. Two large ongoing prospective studies in SSA aim to capture epidemiological data on an unprecedented scale, together 
with surrogate markers of CVD and clinical endpoints. These studies may yield unique insights into the pathogenesis of IHD in HIV infection [39][40].

\section{REFERENCES}

1. Freiberg MS, Chang C-CH, Kuller LH, et al. HIV Infection and the Risk of Acute Myocardial Infarction [Internet]. JAMA Intern. Med. 2013; 173:614-622.

2. Smith CJ, Ryom L, Weber R, et al. Trends in underlying causes of death in people with HIV from 1999 to 2011 (D:A:D): a multicohort collaboration. Lancet (London, England) 2014; 384:241-248.

3. Zhou DT, Kodogo V, Chokuona KFV, et al. Dyslipidemia and cardiovascular disease risk profiles of patients attending an HIV treatment clinic in Harare, Zimbabwe [Internet]. HIV. AIDS. (Auckl). 2015; 7:145-155.

4. $\quad$ Kingery JR, Alfred $Y$, Smart LR,et al. Short and Long Term Cardiovascular Risk, Metabolic Syndrome Prevalence and HIV in Tanzania: A Cross-Sectional Study [Internet]. Heart 2016; 102:1200-1205.

5. Helleberg M, May MT, Ingle SM, et al. Smoking and life expectancy among HIVinfected individuals on antiretroviral therapy in Europe and North America [Internet]. AIDS 2015; 29:221-229.

6. Hatleberg $\mathrm{Cl}$, Ryom L, El-Sadr W, et al. Improvements over time in short-term mortality following myocardial infarction in HIV-positive individuals. AIDS 2016; 30:1583-1596.

*This study shows that improvements in short-term survival after MI appear largley driven by improved medical menagement of CVD risk in HIV positive individuals after MI, emphasising the crucial role of modifiable risk factors.

7. Klein DB, Leyden WA, Xu L,et al. Declining relative risk for myocardial infarction among HIV-positive compared with HIV-negative individuals with access to care. Clin. Infect. Dis. 2015; 60:1278-1280.

8. Silverberg MJ, Leyden WA, Xu L, et al. Immunodeficiency and risk of myocardial infarction among HIV-positive individuals with access to care. J. Acquir. Immune Defic. Syndr. 2014; 65:160-166.

9. Iloeje UH, Yuan Y, L'italien $G$, et al. Protease inhibitor exposure and increased risk of cardiovascular disease in HIV-infected patients. HIV Med. 2005; 6:37-44.

10. Strategies for management of antiretroviral therapy/INSIGHT; D:A:D study groups. Use of nucleoside reverse transcriptase inhibitors and risk of myocardial infarction in HIV-infected patients. AIDS 2008; 22:F17-24.

11. Worm SW, Sabin C, Weber R, et al. Risk of myocardial infarction in patients with HIV infection exposed to specific individual antiretroviral drugs from the $\mathbf{3}$ major drug classes: the data collection on adverse events of anti-HIV drugs (D:A:D) study. J. Infect. Dis. 2010; 201:318-330.

12. Lang $S$, Mary-Krause $M$, Cotte $L$, et al. Impact of individual antiretroviral drugs on the risk of myocardial infarction in human immunodeficiency virus-infected patients: a case-control study nested within the French Hospital Database on HIV ANRS cohort C04. Arch. Intern. Med. 2010; 170:1228-1238.

13. Desai M, Joyce V, Bendavid E, et al. Risk of Cardiovascular Events Associated With Current Exposure to HIV Antiretroviral Therapies in a US Veteran Population 
[Internet]. Clin. Infect. Dis. An Off. Publ. Infect. Dis. Soc. Am. 2015; 61:445-452.

14. INSIGHT START study group, Lundgren JD, Babiker AG, Gordin, F et al. Initiation of Antiretroviral Therapy in Early Asymptomatic HIV Infection [Internet]. N. Engl. J. Med. 2015; 373:795-807.

15. Grinsztejn B, Hosseinipour MC, Ribaudo HJ, et al. Effects of early versus delayed initiation of antiretroviral treatment on clinical outcomes of HIV-1 infection: results from the phase 3 HPTN 052 randomised controlled trial [Internet]. Lancet. Infect. Dis. 2014; 14:281-290.

16. Gaziano TA, Abrahams-Gessel S, Gomez-Olive FX, et al. Cardiometabolic risk in a population of older adults with multiple co-morbidities in rural south africa: the HAALSI (Health and Aging in Africa: longitudinal studies of INDEPTH communities) study [Internet]. BMC Public Health 2017; 17:206.

17. Diaz CM, Segura ER, Luz PM, et al. Traditional and HIV-specific risk factors for cardiovascular morbidity and mortality among HIV-infected adults in Brazil: a retrospective cohort study [Internet]. BMC Infect. Dis. 2016; 16:376.

* This study is the first to evaluate the association between HIV infection and ART exposure with CVD risk in Latin America, and supports resource allocation towards risk factor reduction efforts to prevent CVD events in HIV-infected persons.

18. D:A:D study group. Use of nucleoside reverse transcriptase inhibitors and risk of myocardial infarction in HIV-infected patients enrolled in the D:A:D study: a multicohort collaboration [Internet]. Lancet 2008; 371:1417-1426.

19. Sabin $C A$, Reiss $P$, Ryom $L$, et al. Is there continued evidence for an association between abacavir usage and myocardial infarction risk in individuals with HIV? A cohort collaboration [Internet]. BMC Med. 2016; 14:61.

** This study is particularly relevant because it investigates changes in the association between Abacavir and myocardial infarction, whose mechanism remains unclear after first being described in the DAD cohort in 2008. In particular, the authors ruled out a potential channelling bias effect which influenced the interpretation of the previous study.

20. Vachiat A, McCutcheon K, Tsabedze N, et al. HIV and Ischemic Heart Disease [Internet]. J. Am. Coll. Cardiol 2017; 69:73-82.

21. Wu Z, Huang Z, Jin W, et al. Peripheral Inflammatory Biomarkers for Myocardial Infarction Risk: A Prospective Community-Based Study. Clin. Chem. 2017; 63:663672.

22. Stacey AR, Norris PJ, Qin L, et al. Induction of a Striking Systemic Cytokine Cascade prior to Peak Viremia in Acute Human Immunodeficiency Virus Type 1 Infection, in Contrast to More Modest and Delayed Responses in Acute Hepatitis B and C Virus Infections [Internet]. J. Virol. 2009; 83:3719-3733.

23. Tenorio AR, Zheng $\mathrm{Y}$, Bosch RJ, et al. Soluble Markers of Inflammation and Coagulation but Not T-Cell Activation Predict Non-AIDS-Defining Morbid Events During Suppressive Antiretroviral Treatment [Internet]. J. Infect. Dis. 2014; 210:1248-1259.

24. Kuller LH, Tracy R, Belloso W, et al. Inflammatory and Coagulation Biomarkers and Mortality in Patients with HIV Infection [Internet]. PLoS Med. 2008; 5:e203.

25. Vos AG, Idris NS, Barth RE, et al. Pro-Inflammatory Markers in Relation to Cardiovascular Disease in HIV Infection. A Systematic Review [Internet]. PLoS One 2016; 11:e0147484.

26. Grund B, Baker J V, Deeks SG, et al. Relevance of Interleukin-6 and D-Dimer for Serious Non-AIDS Morbidity and Death among HIV-Positive Adults on Suppressive 
Antiretroviral Therapy [Internet]. PLoS One 2016; 11:e0155100.

27. Bahrami H, Budoff M, Haberlen SA, et al. Inflammatory Markers Associated With Subclinical Coronary Artery Disease: The Multicenter AIDS Cohort Study [Internet]. J. Am. Hear. Assoc. Cardiovasc. Cerebrovasc. Dis. 2016; 5:e003371.

** Large observational study $(\mathrm{N}=923$ ) showing association between inflammatory biomarkers and measures of subclinical coronary artery disease using coronary CTa in an HIV positive population (independent of tradtional risk factors and HIV clinical factors).

28. Alimohammadi M, Pichardo-Almarza C, Agu O, Díaz-Zuccarini V. A multiscale modelling approach to understand atherosclerosis formation: A patient-specific case study in the aortic bifurcation [Internet]. Proc. Inst. Mech. Eng. H. 2017; 231:378-390.

29. Krikke M, Arends JE, Van Lelyveld S, et al. Greater carotid intima media thickness at a younger age in HIV-infected patients compared with reference values for an uninfected cohort. HIV Med. 2017; 18:275-283.

30. Schoffelen AF, de Groot E, Tempelman HA, et al. Carotid Intima Media Thickness in Mainly Female HIV-Infected Subjects in Rural South Africa: Association With Cardiovascular but Not HIV-Related Factors. Clin. Infect. Dis. 2015; 61:1606-1614.

31. D'Ascenzo F, Cerrato E, Calcagno A, et al. High prevalence at computed coronary tomography of non-calcified plaques in asymptomatic HIV patients treated with HAART: a meta-analysis. Atherosclerosis 2015; 240:197-204.

32. Nadel J, O'Dwyer E, Emmanuel S, et al. High-risk coronary plaque, invasive coronary procedures, and cardiac events among HIV-positive individuals and matched controls. J. Cardiovasc. Comput. Tomogr. 2016; 10:391-397.

33. Chistiakov DA, Orekhov AN, Bobryshev Y V. Endothelial Barrier and Its Abnormalities in Cardiovascular Disease. Front. Physiol. 2015; 6:365.

34. Wang T, Yi R, Green LA, et al. Increased Cardiovascular Disease Risk In The HIVPositive Population on ART: Potential Role of HIV-Nef and Tat [Internet]. Cardiovasc. Pathol. 2015; 24:279-282.

35. Torriani FJ, Komarow L, Parker RA, et al. Endothelial Function in HIV-Infected Antiretroviral Naïve Subjects Before and After Starting Potent Antiretroviral Therapy: AIDS Clinical Trials Group Study 5152s [Internet]. J. Am. Coll. Cardiol. 2008; 52:569-576.

36. lantorno $M$, Schar $M$, Soleimanifard $S$, et al.Coronary artery endothelial dysfunction is present in HIV-positive individuals without significant coronary artery disease. AIDS 2017, 31:1281-1289.

* First study to demonstrate asymptomatic HIV positive patents without coronary artery disease (CAD) have signficant endothelial dysfunction which is as severe as HIV negative subjects with established CAD.

37. Lo J, Lu MT, Ihenachor EJ, et al. Effects of Statin Therapy on Coronary Artery Plaque Volume and High Risk Plaque Morphology in HIV-Infected Patients with Subclinical Atherosclerosis: a Randomized Double-Blind Placebo-Controlled Trial [Internet]. lancet. HIV 2015, 2:e52-e63.

38. Longenecker CT, Eckard AR, McComsey GA. Statins to improve cardiovascular outcomes in treated HIV infection [Internet]. Curr. Opin. Infect. Dis. 2016; 29:1-9.

39. Strijdom H, De Boever P, Walzl G, et al. Cardiovascular risk and endothelial function in people living with HIV/AIDS: design of the multi-site, longitudinal EndoAfrica study in the Western Cape Province of South Africa [Internet]. BMC Infect. Dis. 2017, 17:41.

40. Vos A, Tempelman H, Deville W, et al. HIV and risk of cardiovascular disease in sub- 
Saharan Africa: Rationale and design of the Ndlovu Cohort Study. Eur. J. Prev. Cardiol. 2017, doi:10.1177/2047487317702039. 